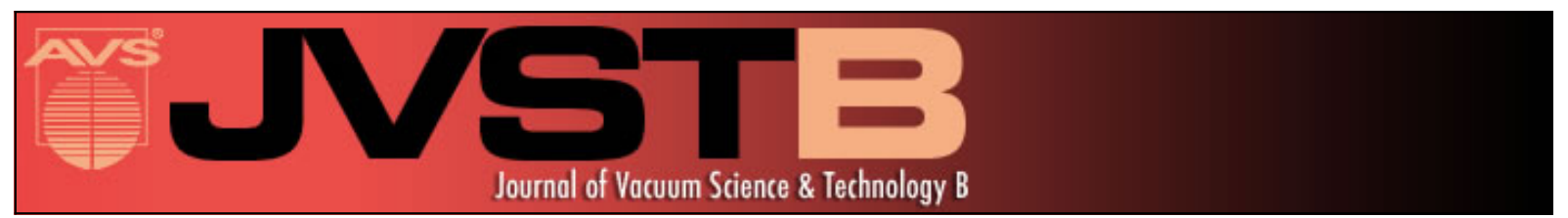

\title{
On the mechanism of improvement of field emission properties of carbon-coated field emitters
}

Toshiharu Higuchi, Masahiro Sasaki, Shota Horie, Yoichi Yamada, Shuji Matsumoto, and Shigeki Fukuda

Citation: Journal of Vacuum Science \& Technology B 33, 03 C102 (2015); doi: 10.1116/1.4903229

View online: http://dx.doi.org/10.1116/1.4903229

View Table of Contents: http://scitation.aip.org/content/avs/journal/jvstb/33/3?ver=pdfcov

Published by the AVS: Science \& Technology of Materials, Interfaces, and Processing

\section{Articles you may be interested in}

Enhanced field emission properties of doped graphene nanosheets with layered SnS2

Appl. Phys. Lett. 105, 043109 (2014); 10.1063/1.4892001

The roles of ruthenium nanoparticles decorated on thin multi-walled carbon nanotubes in the enhancement of field emission properties

Appl. Phys. Lett. 100, 023102 (2012); 10.1063/1.3675460

Improving field emission properties of $\mathrm{GaN}$ nanowires by oxide coating

Appl. Phys. Lett. 94, 243105 (2009); 10.1063/1.3154564

Field emission properties of $\mathrm{N}$-doped capped single-walled carbon nanotubes: A first-principles density-functional study

J. Chem. Phys. 126, 164702 (2007); 10.1063/1.2722750

Enhanced field emission properties of thin-multiwalled carbon nanotubes: Role of Si O x coating J. Appl. Phys. 100, 104303 (2006); 10.1063/1.2384795

WE'RE SEARLHING FOR

SKILLED ANTENNA, RF SYSTEMS AND MICROWAVE DESIGN ENGINEERS.

HELP US ENGINEER A BETTER TOMORROW. LEARN MIRE 


\title{
On the mechanism of improvement of field emission properties of carbon-coated field emitters
}

\author{
Toshiharu Higuchi, ${ }^{a}$ Masahiro Sasaki, Shota Horie, and Yoichi Yamada \\ Institute of Applied Physics, University of Tsukuba, 1-1-1 Tennoudai, Tsukuba, Ibaraki 305-8573, Japan

\begin{abstract}
Shuji Matsumoto and Shigeki Fukuda
Accelerator Laboratory High Energy Accelerator Research Organization, 1-1 Oho, Tsukuba, Ibaraki 305-0801, Japan
\end{abstract}

(Received 13 August 2014; accepted 19 November 2014; published 4 December 2014)

\begin{abstract}
To clarify the origin of the superior field emission characteristics of carbon-coated emitters, the authors investigated the field enhancement and the work function of model systems calculated by numerical simulations. They propose that the field enhancement is due to the triple junctions, which are distributed on the surface of the carbon film consisting of $\mathrm{sp}^{3}$ (diamond-like) insulating and $\mathrm{sp}^{2}$ (graphite-like) conducting nanometer-sized grains. The electric field around the triple junction is one order of magnitude higher than at other places. Based on ab initio density functional theory calculations, the authors found that (1) the work functions of diamond and graphite dramatically decrease down to $3-3.6 \mathrm{eV}$ upon hydrogen termination, and (2) the effective work functions of these models decrease to $2-2.5 \mathrm{eV}$ by applying an external electric field of $2.57 \times 10^{7} \mathrm{~V} / \mathrm{cm}$. They also estimated the field emission current from the potential distribution and the local density of states under the external electric field applied. As a result, the authors found that hydrogen termination significantly increases the field emission current. The results suggest that the triple junction and hydrogen termination are promising candidates as the mechanism of improving the emission of the carbon-coated emitters. (C) 2014 American Vacuum Society.
\end{abstract}

[http://dx.doi.org/10.1116/1.4903229]

\section{INTRODUCTION}

For future vacuum nanoelectronics devices including ultrafast electron sources, compact x-ray sources, lamps, displays, RF-amplifiers, analytical instrument, and high-current density field emitters are required. It has been reported that Fowler-Nordheim analysis of field emission from specific carbon-related materials yields very low effective work functions, even though the materials lack high aspect ratios in their geometrical structures. ${ }^{1,2}$

We have reported that lithographically fabricated $\mathrm{Si}$ FEAs as well as single Si emitters whose tips are coated with an arc-prepared carbon film yield superior field emission features as shown in Fig. 1.,4 Although the tip radius becomes blunt (from 5 to $20 \mathrm{~nm}$ ) upon coating with the carbon film, where geometrical field enhancement should be degraded, the field emission current increases and the slope of the Fowler-Nordheim plot of field emission features becomes reduced upon coating with carbon film. ${ }^{3,4}$ Here, the effective work function is estimated to be $1.7-2.0 \mathrm{eV}$ by using "MAGIC" code. 5

Chung et al. theoretically investigated the field enhancement due to the dielectric around a simple triple junction consisting of metal, dielectric, and vacuum. ${ }^{6}$ They obtained the electric potential near the junction as a function of geometry, dielectric constant of the two-dimensional model. And they found that the key factor to determine the field enhancement is the ratio of the angles subtended by the dielectric and vacuum portions. However, their model is a cylindrical

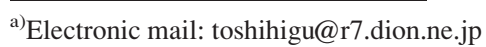

triple junction of metal-dielectric-vacuum model only, and their equation cannot calculate the special distribution of electric field.

Wang et al. calculated the work functions of $\mathrm{H}, \mathrm{OH}, \mathrm{O}$, and NH-terminated graphene by an $a b$ initio Density Functional Theory (DFT) simulation. And they found that the zigzag edge terminated with amine groups has the lowest local work function $(2.0 \mathrm{eV}) .^{7}$ However, their work did not provide the work function under an applied external electric field, let alone estimate the field emission current.

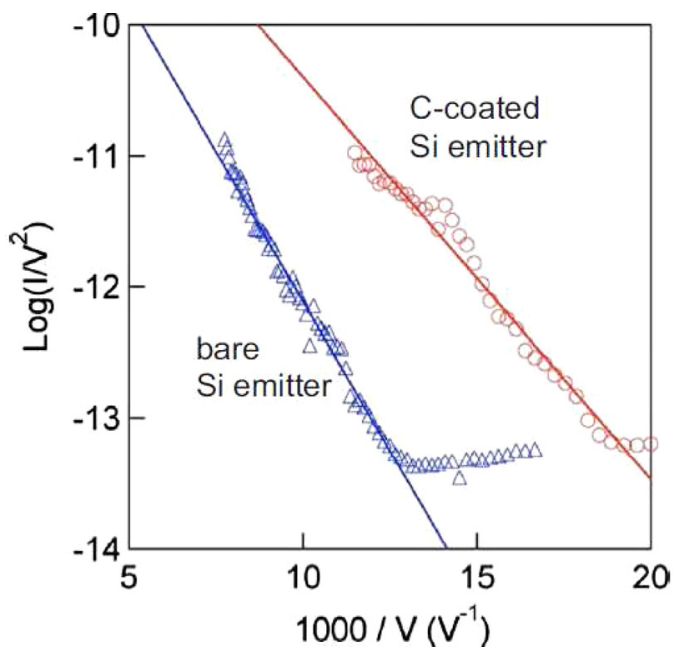

FIG. 1. (Color online) FN plots of C-coated and bare Si emitters obtained from the single emitters. The circles and triangles indicate the carbon coated and bare Si emitters, respectively. The $2 \mu \mathrm{m}$ thick silicon oxide layer is used as a spacer (Ref. 3). 
(a)

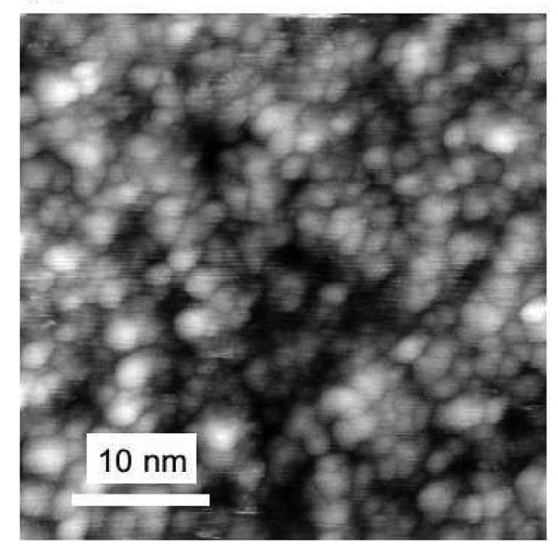

(b)

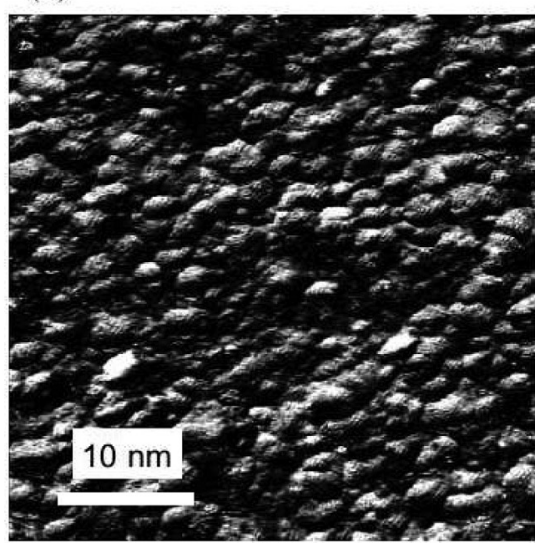

FIg. 2. (a) Constant current STM and (b) LBH images simultaneously obtained from the arc-prepared carbon film $\left(40 \times 40 \mathrm{~nm}{ }^{2}\right.$, Vs $=-1 \mathrm{~V}$, and $\left.\mathrm{I}_{\mathrm{T}}=1 \mathrm{nA}\right)$ (Refs. 3 and 4).

Motivated from the above considerations, in this paper, we investigated the field enhancement near the triple junction point, and work functions on the basis of finite element and $a b$ initio DFT simulations. And we also calculated the emission current distribution by using the model developed by Khazaei. ${ }^{8}$

\section{FIELD ENHANCEMENT AT A TRIPLE JUNCTION}

The scanning tunneling microscopy (STM) and the local tunneling barrier height (LBH) images obtained from the carbon films show the surface consisting of nanometer-scale grains, with no nanoprotrusions having higher aspect ratios that could largely enhance the electric field, as shown in Fig. 2, and the field emission image (FE) obtained shows higher field emission current near the rim of each grain as shown in Fig. 3. ${ }^{3,4}$ These facts suggest that the field enhancement is due to the triple junctions, which are distributed on the surface of the carbon film consisting of $\mathrm{sp}^{3}$ (diamond-like)
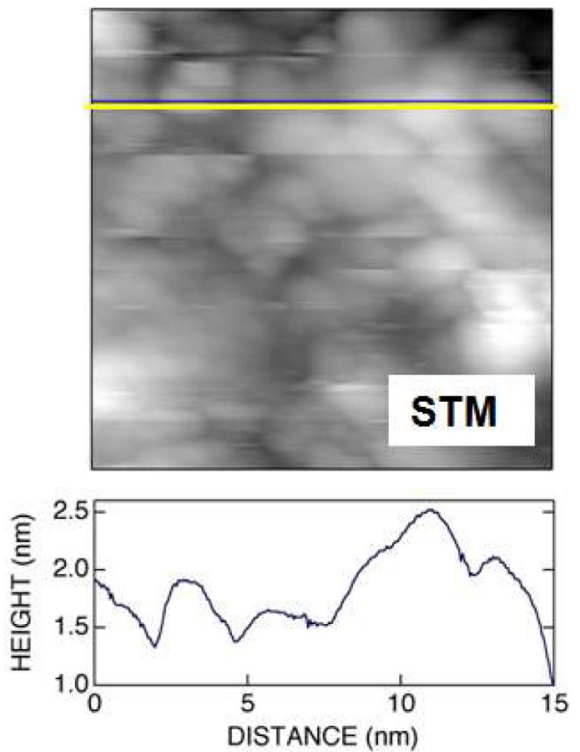

insulating and $\mathrm{sp}^{2}$ (graphite-like) conducting nanometer-sized grains as shown in Fig. 4. Therefore, we considered the dielectric sphere on metal as a simplified model and calculated the electric field. The radii of dielectric spheres are 0.5, 1.0 , and $2.0 \mathrm{~nm}$. The length between the edge of a dielectric sphere and an anode was $5 \mathrm{~nm}$, and the external voltage of $5 \mathrm{~V}$ was applied as shown in Fig. 5 (right). Thus, the electric field in the region of the parallel plate is $1 \times 10^{7} \mathrm{~V} / \mathrm{cm}$.

We have adapted the Poisson Superfish code to calculate the electric field near the triple junction. This code includes a program for calculating static magnetic and electric fields in either two-dimensional Cartesian coordinates or axially symmetric cylindrical coordinates, which was developed by the Los Alamos National Laboratory. ${ }^{9}$ The calculated electric field near the triple junction is one order of magnitude higher than at other places as shown in Fig. 5 (left). Although the relative permittivity $\left(\varepsilon_{\mathrm{r}}\right)$ of the dielectric sphere of the above calculation is 10 (DLC), similar results were obtained in both $\varepsilon_{r}=20$ and $\varepsilon_{r}=5$ (diamond).
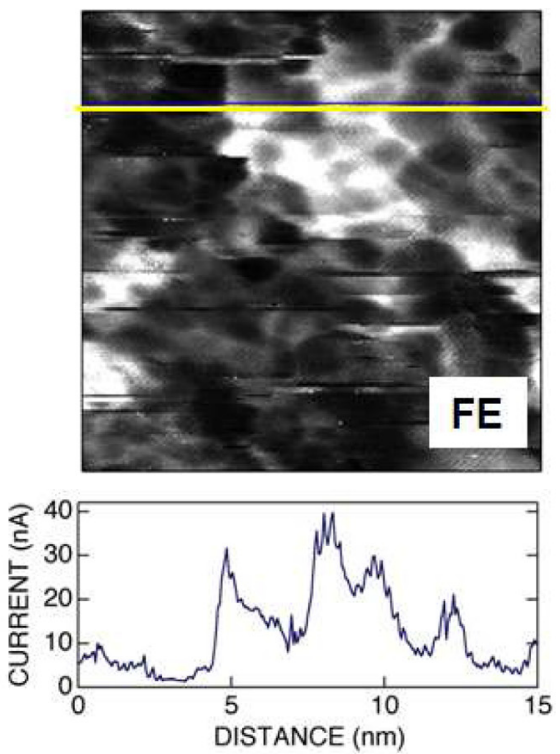

FIG. 3. (Color online) Constant current STM and FE images simultaneously obtained from the arc-prepared carbon film $\left(15 \times 15 \mathrm{~nm}{ }^{2}\right.$, Vs $(\mathrm{STM})=-3.7 \mathrm{~V}$, $\left.\mathrm{I}_{\mathrm{T}}=1 \mathrm{nA}, \mathrm{Vs}(\mathrm{FE})=-5.5 \mathrm{~V}\right)($ Refs. 3 and 4$)$. 


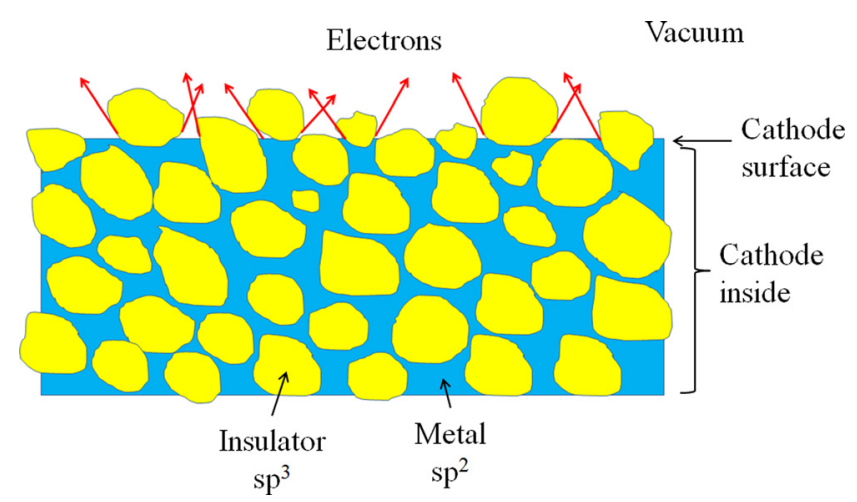

FIG. 4. (Color online) Triple junction model. Nanometer-sized $\mathrm{sp}^{3}$ insulator clusters embedded on and in the surrounding $\mathrm{sp}^{2}$ matrix.

Many electrons emitted on the cathode surface near the triple junction can penetrate through the nanometer sized insulator, because electron inelastic mean free paths in solid ${ }^{10}$ are (1) $6 \mathrm{~nm}$ at $5 \mathrm{eV}$ and (2) more than $100 \mathrm{~nm}$ at $1 \mathrm{eV}$. Thus, the triple junction model is consistent with the observed FE image obtained from STM and it is a promising candidate as the emission improvement mechanism of the carbon-coated emitters.

\section{AB INITIO DFT CALCULATION}

The work functions, and the field emission current as well as emission patterns of carbon-related materials under applied external fields were calculated by using QuantumESPRESSO (QE), developed by the DEMOCRITOS National Simulation Center in Trieste, Italy. ${ }^{11}$ This program is an integrated suite of computer codes for electronicstructure calculations and the nanoscale modeling of materials. It is based on density-functional theory, plane waves, and pseudopotentials.

Calculations were made for clean and hydrogenterminated (H-terminated) diamond and graphite as shown in Fig. 6. Electrons emit from diamond $(001)-(1 \times 1)$ and graphite zigzag edge, respectively. The diamond and graphite had film thicknesses of $1.075 \mathrm{~nm}$ and $1.579 \mathrm{~nm}$, respectively. These thicknesses are nearly the same as the grain size of the carbon films enhancing field emission as shown in Fig. 2. The H-terminated diamond and H-terminated

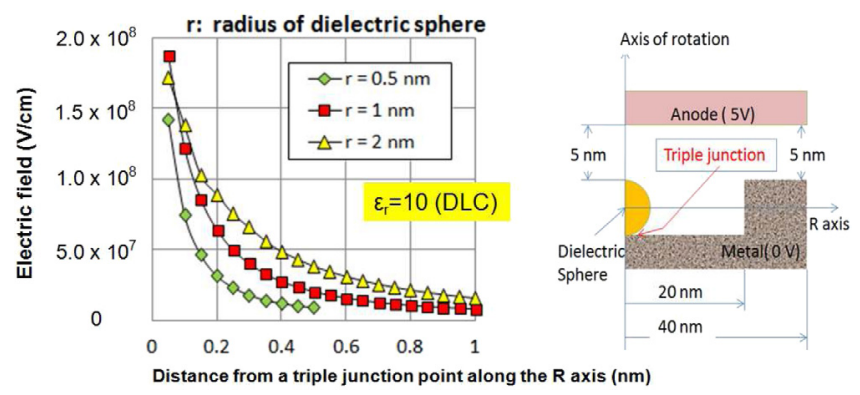

FIG. 5. (Color online) Calculated triple junction model (right) and calculated electric field near the triple junction (left) as calculated by Poisson Superfish. Strong electric field enhanced near the triple junction of the dielectric sphere $\left(\varepsilon_{r}=10\right)$ on metal. The diamonds, squares, and triangles indicate the radius of dielectric sphere, $r=0.5,1,2 \mathrm{~nm}$, respectively.

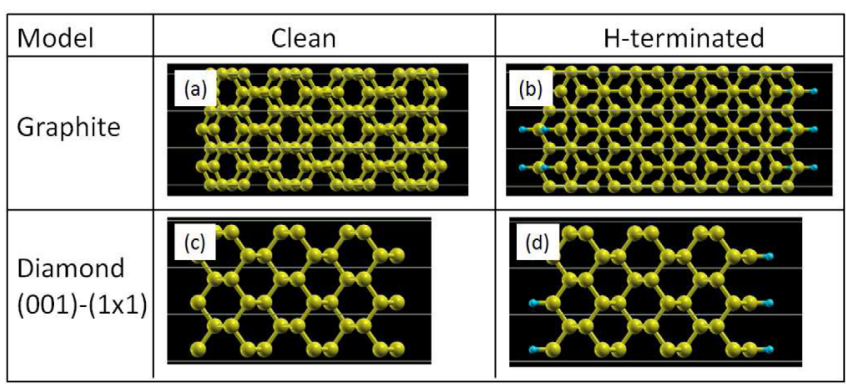

FIG. 6. (Color online) Calculated models for (a) clean graphite (zigzag edge); (b) H-terminated graphite (zigzag edge); (c) clean diamond (001)$(1 \times 1)$; and $(\mathrm{d}) \mathrm{H}$-terminated diamond $(001)-(1 \times 1)$.

graphite had C-H bond lengths of 0.110 and $0.101 \mathrm{~nm}$, respectively. These bond lengths were obtained from O'Donnell's and Miyamoto's reports. ${ }^{12,13}$ It is well known that the equilibrium atomic positions on a crystal surface are generally different from those on an ideal bulk-terminated surface. Here, a structural relaxation was taken into account. The cutoffs were $40 \mathrm{Ry}$ for the wave functions and $480 \mathrm{Ry}$ for the charge density, and a $\mathrm{k}$ mesh $(10 \times 10 \times 1)$ was used. We calculated electronic properties under an external electric field applied. The form of external electric field is a saw-tooth like.

Figure 7 shows the calculated work functions where (1) the work functions (without external electric field) of diamond and graphite dramatically decrease to $3-3.6 \mathrm{eV}$ upon hydrogen termination and (2) the effective work functions of the H-terminated models decrease to $2-2.5 \mathrm{eV}$ by applying an external electric field of $2.57 \times 10^{7} \mathrm{~V} / \mathrm{cm}$.

Next, we calculated the field emission current using the model developed by Khazaei based on the Penn-Plummer model. ${ }^{8,14}$ Equation (1) is the Khazaei's equation. Here, we give an outline of this equation. The details are described in his paper ${ }^{8}$

$$
j_{i}(\varepsilon)=\frac{2 e \hbar}{m_{e}} \cdot f(\varepsilon) \cdot S_{i} \cdot \lambda_{i}^{-2}(\varepsilon) \cdot D_{i}^{2}(\varepsilon) \cdot g_{i}\left(\varepsilon, x_{l, i}\right),
$$

$j_{i}(\varepsilon)$ is the field emission current along the grid line $i$. In $a b$ initio calculations, the supercell is generally discretized by introducing a fine grid. Thus, the total current $j(\varepsilon)$ is

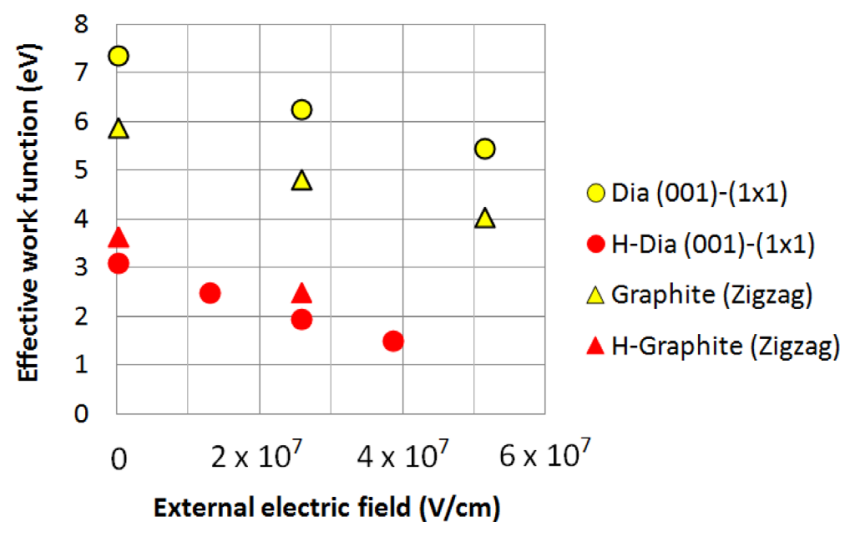

FIG. 7. (Color online) Effective work functions vs external electric field for diamond and graphite as calculated by Quantum-ESPRESSO. 
calculated to be the sum of the currents along the grid line $i$; $j(\varepsilon)=\sum_{i} j_{i}(\varepsilon)$. In Eq. (1), $m_{e}$ is the electron effective mass, $\hbar$ is the reduced Plank constant, and $\varepsilon$ is the electronic state with energy. $f(\varepsilon)$ is the Fermi-Dirac distribution, and $S_{i}$ is the area of the surface element $i . \lambda_{i}(\varepsilon)$ is a slowly varying function of energy resulting from the asymptotic matching of the wave function of emitting state at the left turning point $x_{l, i}$ with WKB wave function inside the barrier. The left and right turning points, $x_{l, i}$ and $x_{r, i}$, along the grid line $i$ are determined as the points where the energy of the emitting state becomes equal with the potential barrier energy $u_{i}(x)$, with $x$ being the coordinate along the emission direction. $D_{i}^{2}(\varepsilon)$ indicates the probability of electron tunneling through the nanostructure-vacuum barrier $u_{i}(x)$, and $g_{i}\left(\varepsilon, x_{l, i}\right)$ is the local density of states (LDOS) at the left turning point. $\lambda_{i}(\varepsilon)$ is given by

$$
\lambda_{i}(\varepsilon)=(\pi / 3)^{\frac{1}{2}} \cdot\left(c_{i} / 3\right)^{-\frac{1}{3}}\left[\Gamma\left(\frac{2}{3}\right) \cos (\pi / 6)\right]^{-1},
$$

where $c_{i}$ is obtained by fitting the effective potential $\frac{2 m_{e}}{\hbar^{2}}\left[u_{i}(x)-\varepsilon\right]$ to $c_{i}^{2}\left(x-x_{l, i}\right)$ at the left turning point, and $\Gamma$ is the gamma function. The tunneling probability $D_{i}^{2}(\varepsilon)$ is expressed as

$$
D_{i}^{2}(\varepsilon)=\exp \left[-2 \sqrt{\frac{2 m_{e}}{\hbar^{2}}} \int_{x_{l, i}}^{x_{r, i}} \sqrt{u_{i}(x)-\varepsilon} d x\right] .
$$

Within above approach, the necessary data for calculating emission currents $j_{i}(\varepsilon)$, the LDOS $g_{i}\left(\varepsilon, x_{l, i}\right)$, the tunneling probability $D_{i}^{2}(\varepsilon)$, and the slowly varying function of energy $\lambda_{i}(\varepsilon)$ are calculated by using the output data of the QE.

In the actual calculation, we use these values: (1) $2 e \hbar / m_{e}=2.3153 \times 10^{-4} \mathrm{CeVs} / \mathrm{kg}\left(m_{e}\right.$ is the electron effective mass, but we assumed it to be the rest mass here.). (2) $f(\varepsilon)=0.5$ (as temperature is $0 \mathrm{~K}$ ). (3) $S_{i}$ is a grid mesh size (in $\AA^{2}$ ) of the face perpendicular to the emission
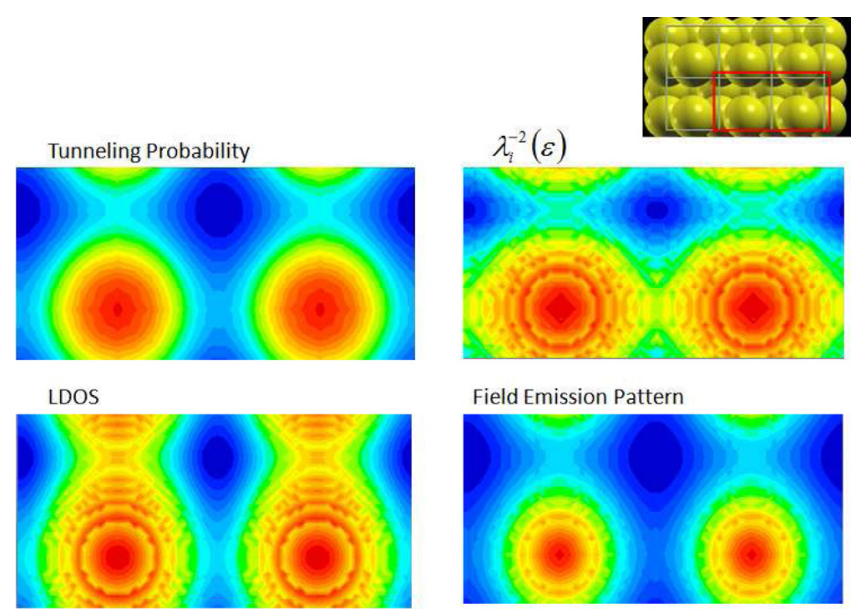

FIG. 8. (Color online) Calculated patterns of the probability of electron tunneling through a nanostructure-vacuum barrier, $\lambda_{i}^{-2}(\varepsilon)$, the LDOS, and field emission of the clean diamond $(001)-(1 \times 1)$. Inset shows the top view of an atomic structure model. The inside of the red frame of inset corresponds to the frame of calculated figures. direction. (4) $c_{i}$ is obtained by fitting the potential distribution at Fermi-energy (in eV) of the left turning point. The potential distribution is the output data of the QE calculation. (5) In this calculation, we defined $\varepsilon=$ Fermi energy. $u_{i}(x)$, in $\mathrm{eV}$, is obtained by using the potential distribution, which is the output of the QE calculation. (6) $g\left(\varepsilon, x_{l, i}\right)$, in $\mathrm{eV}^{-1} \AA^{-3}$ is obtained by the output data of QE: local density of states at the Fermi energy.

Figures 8 and 9 show the calculated distributions of the probability of electron tunneling through a nanostructurevacuum barrier, $\lambda_{i}^{-2}(\varepsilon)$, the LDOS, and field emission. Figure 8 is for the case of the clean diamond $(001)-(1 \times 1)$. From this figure, we found that $(1)$ current density is the highest on the top of the carbon atom and (2) the patterns of the probability, $\lambda_{i}^{-2}(\varepsilon)$, and LDOS are corresponding to emission pattern. Figure 9 is the case of $\mathrm{H}$-terminated diamond $(001)-(1 \times 1)$. From this figure, we found that (1) more electrons are released from the inbetween position of hydrogen atoms and (2) the patterns of the probability does not correspond to LDOS and to emission pattern.

Although the calculations described above were the case of the clean diamond and $\mathrm{H}$-terminated diamond, similar results were obtained from the case of the clean graphite and H-terminated graphite, i.e., more electrons are released from the in-between position of hydrogen atoms in the cases of $\mathrm{H}$-terminated graphite, although the current density of the clean graphite is most high on the top of the carbon atom.

Table I lists the effective work functions and total field emission current $j(\varepsilon)=\sum_{i} j_{i}(\varepsilon)$ at applied external electric field of $2.57 \times 10^{7} \mathrm{~V} / \mathrm{cm}$. From this table, we found that hydrogen termination significantly increases the emission current: by fourteen orders of magnitude for diamond (001) and by seven for graphite.

From above results, we conclude that hydrogen termination dramatically decreases the work functions and increases the field emission current.
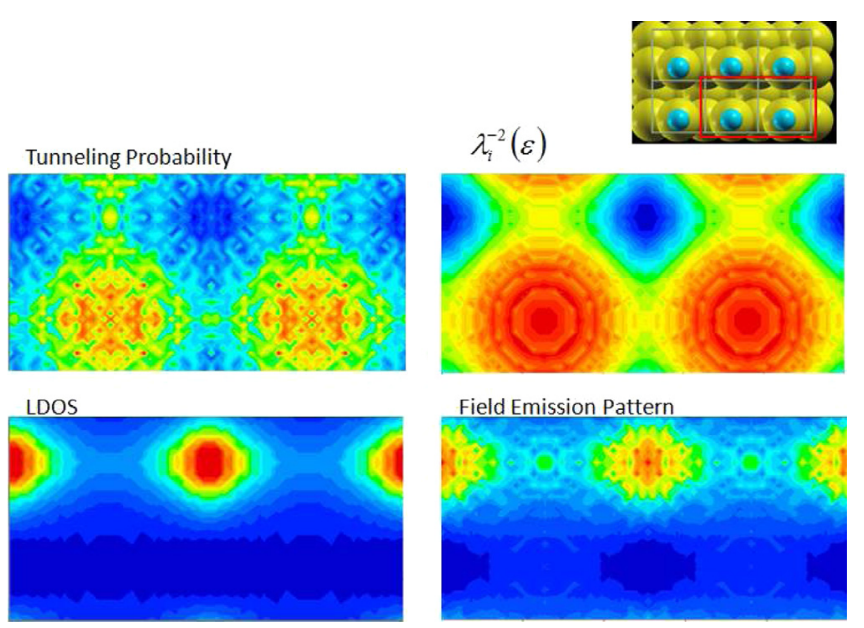

FIG. 9. (Color online) Calculated patterns of the probability of electron tunneling through a nanostructure-vacuum barrier, $\lambda_{i}^{-2}(\varepsilon)$, the LDOS, and field emission of the H-terminated diamond $(001)-(1 \times 1)$. Inset shows the top view of an atomic structure model. The inside of the red frame of inset corresponds to the frame of calculated figures. 
TABLE I. Effective work functions and field emission current at applied external field of $2.57 \times 10^{7} \mathrm{~V} / \mathrm{cm}$.

\begin{tabular}{lcc}
\hline \hline Model & $\begin{array}{c}\text { Effective work } \\
\text { function }(\mathrm{eV})\end{array}$ & $\begin{array}{c}\text { Field emission } \\
\text { current }\left(\mathrm{A} / \mathrm{m}^{2}\right)\end{array}$ \\
\hline Diamond $(001)-(1 \times 1)$ & 6.25 & $1.57 \times 10^{-8}$ \\
H-Diamond $(001)-(1 \times 1)$ & 1.96 & $8.70 \times 10^{6}$ \\
Graphite (zigzag) & 4.81 & $4.69 \times 10^{-2}$ \\
H-Graphite (zigzag) & 2.49 & $7.15 \times 10^{5}$ \\
\hline \hline
\end{tabular}

\section{DISCUSSIONS}

First, we discuss the reason why hydrogen termination significantly increases the emission current by fourteen orders of magnitude for diamond $(001)-(1 \times 1)$. Field emission current depend on the product of the tunneling probability, $\lambda_{i}^{-2}$, and LDOS. The $\lambda_{i}^{-2}$ of the H-terminated is the same as that of the clean. The mean value of LDOS of the clean is $1.65 \times 10^{-2} \mathrm{eV}^{-1} \AA^{-3}$. In contrast, its value of the $\mathrm{H}$ terminated is $5.5 \times 10^{-5}$, which is three orders of magnitude smaller than that of the clean. The mean value of the tunneling probability for the clean is $4.4 \times 10^{-23}$. However, the mean value for the $\mathrm{H}$-terminate is $1.0 \times 10^{-5}$, which is 18 orders of magnitude larger than that of the clean. This is the reason why the emission current of the $\mathrm{H}$-terminated is larger than that of the clean.

Second, we discuss the reason why many electrons are released from the in-between position of hydrogen atoms in the H-terminated diamond, although the current density of the clean is highest on the top of the carbon atom. The maximum and minimum tunneling probabilities of the clean are $9.0 \times 10^{-23}$ and $2.1 \times 10^{-23}$, respectively; the ratio of the maximum to the minimum is 4.3 . On the other hand, in the case of the H-terminated, $\max .=1.8 \times 10^{-5}$ and min.$=4.4 \times 10^{-6}$; the ratio is 4.1 . Thus, both the ratios are quite similar. However, the maximum and minimum LDOS of the clean are $8.3 \times 10^{-2}$ and $2.1 \times 10^{-2}$, respectively; the ratio is 2.6. In the case of the H-terminated, max. $=3.9 \times 10^{-4}$ and $\min .=3.3 \times 10^{-6}$; the ratio is 120 . It means that the LDOS of the H-terminated are concentrated in between position of hydrogen atoms. This is the reason why many electrons are released from the in-between position of hydrogen atoms in the case of $\mathrm{H}$-terminated diamond.

\section{SUMMARY AND CONCLUSIONS}

From the field enhancement analyses around triple junction, and the work function and emission current analyses based on the ab initio DFT calculation, we found that (1) the calculated electric field near the triple junction is one order of magnitude higher than at other places, (2) the work functions of diamond and graphite dramatically decrease to $3-3.6 \mathrm{eV}$ upon hydrogen termination, and the effective work functions of the H-terminated models decrease to $2-2.5 \mathrm{eV}$ by applying an external electric field of $2.57 \times 10^{7} \mathrm{~V} / \mathrm{cm}$, (3) many electrons are released from the in-between position of hydrogen atoms in the cases of $\mathrm{H}$-terminated diamond and graphite, although the current densities of the clean diamond and graphite are highest on the top of the carbon atom, and (4) hydrogen termination significantly increases emission current: by 14 orders of magnitude for diamond $(001)-(1 \times 1)$ and by seven for graphite.

From these investigations, we conclude that the triple junction model and the hydrogen terminated model are promising candidates as the mechanism of improving the field emission feature upon coating with the arc-prepared carbon film.

\section{ACKNOWLEDGMENTS}

The authors wish to thank H. Mimura of Shizuoka University and M. Khazaei from the National Institute for Material Science for their helpful discussions.

${ }^{1}$ R. G. Forbes, Solid-State Electron. 45, 779 (2001).

${ }^{2}$ R. G. Forbes and J. P. Xanthakis, Surf. Interface Anal. 39, 139 (2007).

${ }^{3}$ S. Nagashima, S. Fujita, K. Adachi, Y. Yamada, and M. Sasaki, J. Vac. Sci. Technol., B 28, C2A13 (2010).

${ }^{4}$ S. Horie, K. Asanagi, T. Higuchi, Y. Yamada, and M. Sasaki, Proceedings of the 20th International Display Workshops (IDW'13), Sapporo, Japan, 4-6 December 2013 (The Institute of Image Information and Television Engineers, The Society for Information Display, 2013), p. 839.

${ }^{5}$ MAGIC Tool Suite, FDTD-PIC Software for EM Design and Simulation, ATK. COM.

${ }^{6}$ M. S. Chung, S. C. Hong, P. H. Cutler, N. M. Miskovsky, B. L. Weiss, and A. Mayer, J. Vac. Sci. Technol. 24, 909 (2006).

${ }^{7}$ W. Wang, J. Shao, and Z. Li, Technical Digest of IVNC, 25th International Vacuum Nanoelectronics Conference, Jeju, Korea, 9-13 July 2012.

${ }^{8}$ M. Khazaei, A. A. Farajian, and Y. Kawazoe, Phys. Rev. Lett. 95, 177602 (2005).

${ }^{9}$ See http://laacg1.lanl.gov/laacg/services/download_sf.phtml.

${ }^{10}$ M. P. Seah and W. A. Dench, Surf. Interface Anal. 1, 2 (1979).

${ }^{11}$ Quantum Espresso, see http://www.quantum-espresso.org/.

${ }^{12}$ K. M. O’Donnell, T. L. Martin, N. A. Fox, and D. Cherns, Phys. Rev. B 82, 115303 (2010).

${ }^{13}$ Y. Miyamoto, K. Nakada, and M. Fujita, Phys. Rev. B 59, 9858 (1999).

${ }^{14}$ D. R. Penn and E. W. Plummer, Phys. Rev. B 91216 (1974). 